\title{
Conservative Management of a Distal Epiphyseal Metacarpal Fracture in a Skyros Pony
}

\author{
Eleni-Maria AMANITI, Nikolaos DIAKAKIS*, Michail PATSIKAS and Ioannis SAVVAS \\ Companion Animal Clinic, Department of Clinical Studies, School of Veterinary Medicine, Aristotle University \\ of Thessaloniki, 11 Voutyra Str., 54627 Thessaloniki, Greece
}

Epiphyseal fracture, also known as epiphysiolysis, is the loosening or separation, either partial or complete, of an epiphysis from the shaft of a long bone. Distal epiphyseal fractures in foals pose a substantial challenge due to their guarded prognosis. This report describes the clinical signs, diagnosis, successful conservative treatment of an 1-month-old, male Skyros pony with an epiphyseal fracture of the right third metacarpal bone (type II SalterHarris). The owner declined surgical treatment because of financial considerations along with the unfavorable prognosis. Conservative fracture treatment was pursued through external reduction and coaptation. The total duration of the conservative treatment was eighteen weeks and the foal returned to complete health.

Key words: epiphysiolysis, pony

\author{
J. Equine Sci. \\ Vol. 19, No. 3 \\ pp. 57-61, 2008
}

Epiphyseal fracture, also known as epiphysiolysis, is the loosening or separation, either partial or complete, of an epiphysis from the shaft of a long bone $[6,9]$. Epiphyseal injury is likely to occur, since the cartilaginous growth plate is weaker than the surrounding bone and it is replaced with trabecular bone. In general, the further along the limb the physis is located, the sooner in life it will become functionally inactive [1]. Fractures involving the physis of a bone may be classified according to Salter-Harris, based upon the configuration and relationship of the fracture plane to the growing cells of the metaphyseal growth plate [4].

Epiphysiolysis is common and documented in a variety of species like dogs, cats, cows and horses [12]. Distal physeal fractures of the third metacarpal are common injuries in suckling and weanling foals, and may be caused by external trauma, fall or kick $[1,12]$. The fact that the metacarpal region is only protected by skin explains the frequency of open fractures, which are further complicated by injuries of the sheath of digital tendons and the suspensory ligament.

Clinical signs of epiphysiolysis are often obvious and

This article was accepted March 14, 2008

*Corresponding author. e-mail: diakakis@vet.auth.gr differential diagnosis is easy to make. Still, radiographs are important to assess the type and the degree of displacement of the fracture [7]. Distal epiphyseal fracture of the third metacarpal bone poses a substantial challenge for recovery, either treated conservatively or surgically [12]. The substantial expense of surgery and the risk of failure dissuades owners from pursuing repair. An unsuccessful outcome in foals may be the result of infection and non-union or secondary complications such as cast sores [1]. Therefore, distal epiphyseal fractures in foals pose a substantial challenge due to their guarded prognosis.

This report describes the clinical signs, diagnosis and successful conservative treatment of an 1-month-old, male Skyros pony with a epiphyseal fracture of the right third metacarpal bone.

An 1-month-old, male Skyros pony, weighing $60 \mathrm{~kg}$, was presented at the Companion Animal Clinic of School of Veterinary Medicine, Aristotle University of Thessaloniki, Greece, with a history of an acute (oneday-old), non-weight-bearing lameness of the right front limb.

Clinical evaluation revealed a warm, oedematous and swollen distal right metacarpal region. Crepitation and pain around the right fore fetlock area was evident on 


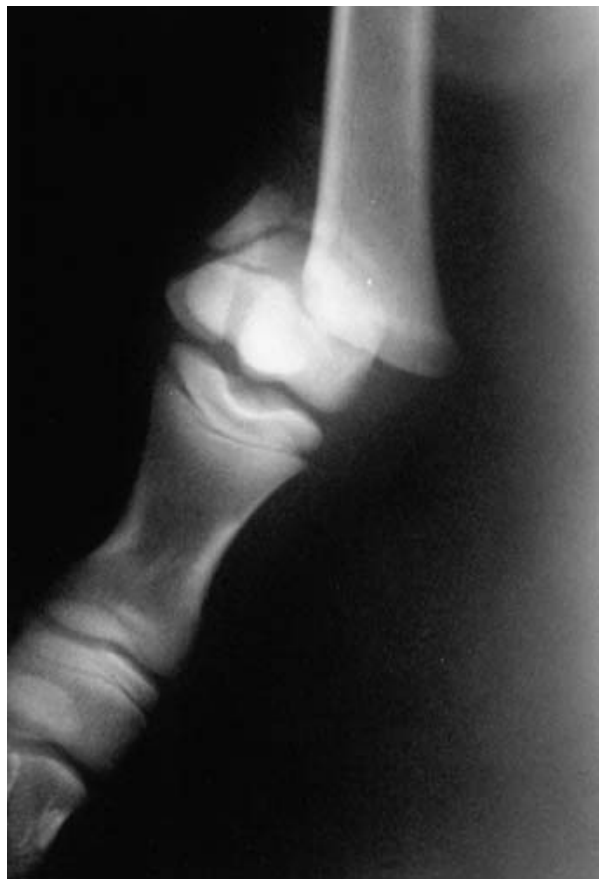

(a)

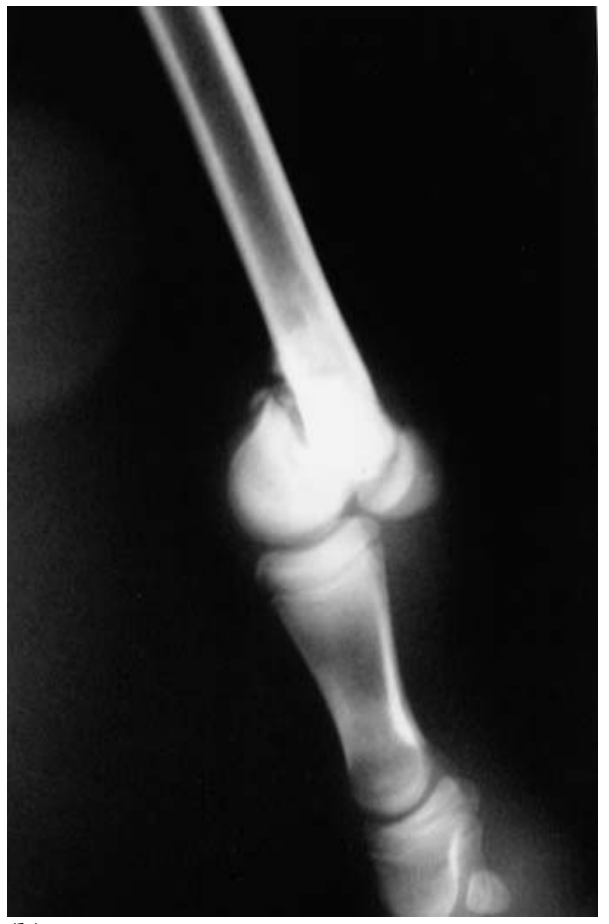

(b)

Fig. 1. Dorsopalmar (a) and mediolateral (b) radiographs of the right metacarpophalangeal (fetlock) joint of an 1-month-old Skyros pony showing a type II Salter-Harris fracture of the distal 3rd metacarpal physis. The proximal part was displaced mediopalmarly and the distal part was rotated dorsally resulting in overriding of the fragmented parts, malposition of the condyles of the third metacarpal bone and distal displacement of the proximal sesamoid bones.

palpation, suggestive of a distal epiphyseal fracture. No further abnormalities were found and the foal was bright, alert and responsive.

Plain radiographs (dorsopalmar, mediolateral, dorso $45^{\circ}$ lateral-palmaromedial oblique, dorso $45^{\circ}$ medialpalmarolateral oblique) revealed a closed, new, type II Salter-Harris fracture of the distal third metacarpal bone (Fig. 1).

The owner declined surgical treatment because of financial considerations along with the unfavorable prognosis.

Conservative management was pursued through external reduction and coaptation. Preanesthetic preparation included a thorough physical examination, examination of cardiovascular status, including ancillary tests such as hematocrit and plasma protein determination. The foal was sedated with romifidine (60 $\mu \mathrm{g} / \mathrm{kg}$ body weight). Local anesthesia of the distal limb was achieved with intravenous administration of xylocaine ( $5 \mathrm{ml}, 2 \%$ xylocaine) through the cephalic vein, following the cranial application of an Ershmach bandage. Moreover, antitetanic toxoid (3,000 IU, intramuscularly) was administered to the foal.

Closed reduction was achieved manually. More specifically, the pastern was pulled distally, and the fetlock dorsally. The two parts of the fragment were manipulated so as to be aligned. In order to immobilize the fracture, a fiberglass cast was used $\left(\right.$ Vetcast $\left.^{\circledR}\right)$. The carpus and the foot were incorporated in the cast. Prior to the application of the cast, two layers of stockinette $\left(\right.$ Seton $^{\circledR}$ ) and padding (Softban ${ }^{\circledR}$ ) were applied to prevent pressure sores on the palmar aspect of the carpus and fetlock. Ketoprofen $(1 \mathrm{mg} / \mathrm{kg}$, once daily, intravenously for 5 days), penicillin (10,000 IU $/ \mathrm{kg}$, once daily for 5 days, intramuscularly) and streptomycin (15 mg/kg once daily intramuscularly for 5 days) were administered to the foal.

The cast was examined daily for evidence of rubbing, discharge or cracking. Moreover, the limb was examined thoroughly for signs of skin damage or necrosis, changes in weight-bearing position, edema 


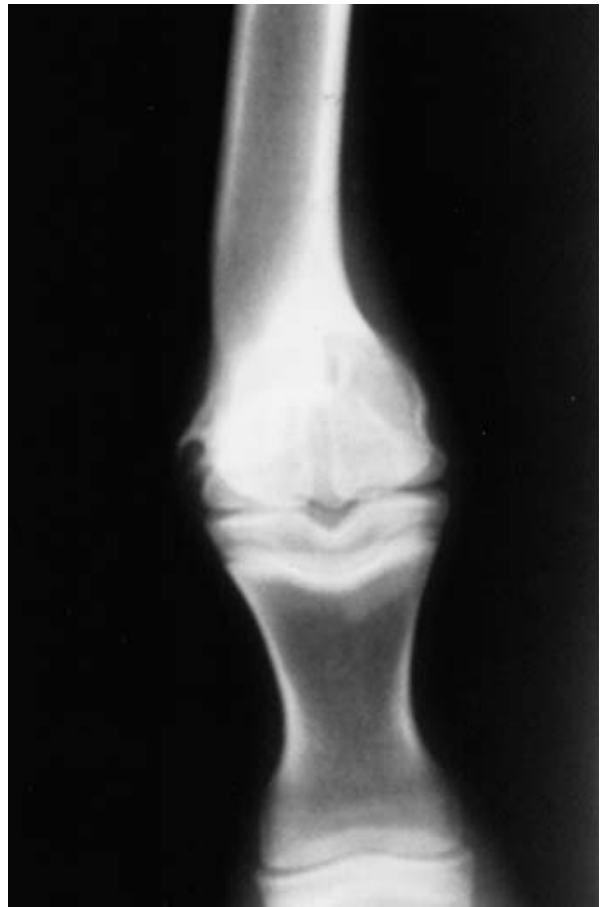

(a)

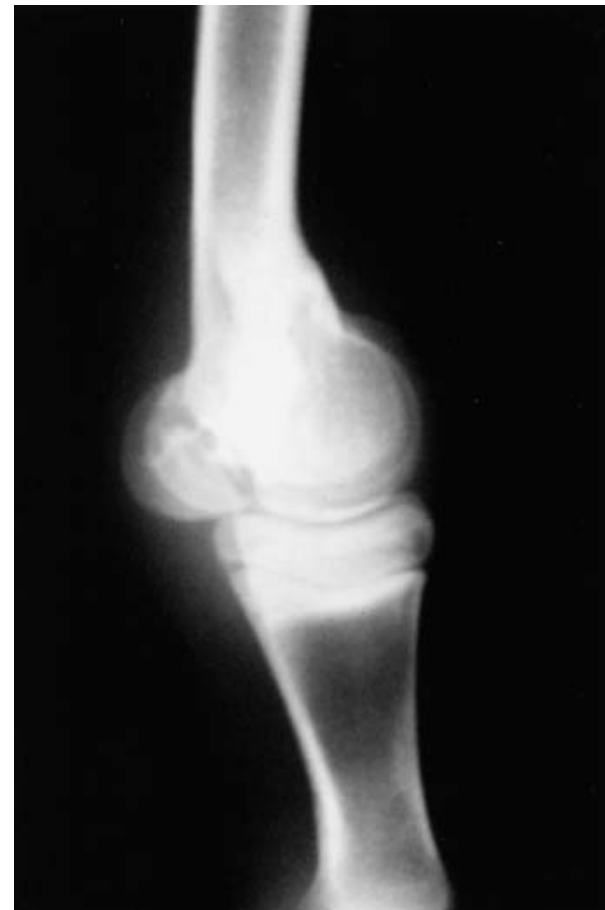

(b)

Fig. 2. Dorsopalmar (a) and lateromedial (b) radiographs of the right fetlock 1 month after the application of the Robert-Jones bandage ( 2 months after fracture incidence) demonstrated the remodeling process of the fracture characterized by disappearance of the fracture line and development of the trabecular pattern within the callus. Mild rotation of the third metacarpal shaft and osteoporosis of the involved bones were evident. The fragmented segments were still malpositioned.

above the proximal cast end or wet spots on the cast. The cast was fitting well and the foal was comfortable, so it was left on for four weeks. One month after cast application, a latero-medial radiographic view was taken, so as to estimate fracture healing. A bony callus formation had bridged the gap between the fragmented segments; however they remained in malposition. At that time the foal was showing minimal lameness $(1 / 5)$ at walk. Based on the clinical and the radiological findings a decision was made to replace the cast with a Robert-Jones bandage reinforced with a splint.

The Robert-Jones bandage extended from the hoof to the elbow. The cotton wool was compressed with gauze bandages. The Robert-Jones bandage was finally reinforced with a wrap-around splint. Eight weeks after the initial injury, a latero-medial and dorsopalmar radiographic view were taken which demonstrated the remodeling process of the fracture characterized by disappearing of the fracture line and development of the trabecular pattern within the callus (Fig. 2). Despite the fact the fragmented segments were still misaligned the foal was sound at that time. The splint was removed and the Robert-Jones bandage was changed for a lighter, support bandage. The bandage was removed 12 weeks after the initial injury and the foal returned to complete health. During those four weeks the pony was kept confined in a box. After the removal of the cast, although the total length of the limb was normal, the foal presented with dropped fetlock, hyperextension of the hoof due to the weakening of the musculotendinous flexor and extensor units. In order for the limb to regain its strength, the foal was moved to a small paddock where it could move around at walk and trot, but not canter. Moreover, daily, it was put on a gradually increasing hand-walking exercise program, along with its mother. That gradual exercise program lasted till the 18th week after the injury. At that time the foal was clinically normal. 
The immature long bone is divided into four distinct regions: the physis, the epiphysis, the metaphysis and the diaphysis [9]. Injury to the cartilage layers of the physis, even if it is common, during and after fracture and during the repair, often results in premature closure with several disturbances in longitudinal bone growth $[6,11]$. If the injury involves a physis that has minimal residual growth left (such as the distal physis of the third metacarpal bone) then the potential for interference with growth is probably negligible [1]. Alignment of the limb is critical during the repair, and closure of the distal growth plate to prevent asymmetric growth should be a goal in the repair [1].

Epiphysiolysis can result from any external trauma. The cause is often the result of the dam having stepped on the foal's limb [2]. The diagnosis of a fracture of the third metacarpus can usually be made from the clinical signs. Horses with such fractures present with an acute non-weight-bearing lameness [13]. The veterinarian should very carefully examine and manipulate the limb before assessing the horse's gait, as further damage can be caused by moving the horse. The affected limb is frequently unstable and crepitation can be felt. Depending on the duration of the injury, swelling may or may not be present [13]. A tentative diagnosis of a fracture can be made based on the non-weight-bearing lameness and the palpable instability [13].

Radiographs are necessary for characterizing the configuration of the fracture. The joints above and below the fracture should always be included in order to evaluate the integrity of the articular surfaces [9]. Four views should be obtained: the lateromedial, the dorsopalmar or dorsoplantar, and two oblique projections [11]. Radiographic diagnosis is not difficult provided good-quality radiographs are obtained [12]. There are several treatment options for third metacarpal fractures. Factors that play a role in the treatment method include age, weight, sex, temperament, value, and intended use of the animal, as well as fracture configuration, presence of vascular insult, and whether the fracture is open or closed [5].

Although reduction and fixation of third metacarpal region fractures present no great technical difficulties, the prognosis for a successful outcome remains guarded to poor especially when surgery is the chosen treatment [7].

In foals, even if most third metacarpal fractures can be repaired surgically, physeal fractures represent a special condition, and although the limited body weight of foals and yearlings allows rigid internal fixation, the epiphyseal portion is generally quite small and accommodates only a limited number of screws [1, $11,12]$. In young foals, older than six weeks or heavier or with marked instability, the fracture must be repaired with compression screws through the metaphyseal component in addition to external coaptation [12]. Young foals, less than six weeks old, can be treated with external reduction, which may be difficult owing to muscle contraction, and cast coaptation for two to three weeks followed by two to three weeks in a splinted bandage $[5,12]$. As in the case presented in this study, a rapid immobilization of the fracture can have a favorable prognosis without general anesthesia. Selection of the cast material is important. In this case external coaptation using a fiberglass cast was chosen, because it allows the skin to breathe, making it more comfortable for the foal. Fiberglass is light in weight, and the cast was well tolerated by the foal, so it was not necessary to change it at 10-14 days intervals. The limb was evaluated daily so as to promptly deal with any complications. Extra care was taken for impingements on soft-tissue structures or skin traumas that would signal the need for cast removal $[1,5,11-13]$.

In foals, the leg contralateral to the fractured leg should always be well supported with a Robert-Jones type of bandage to prevent excessive stresses being placed on the supportive tendons and ligaments [5]. In this case, although no such precaution was taken, no complications were observed.

Healing of the bone requires 2-3 months in foals, depending on the complication and severity of the fracture. Stall rest should be anticipated for four months in foals, although hand walking can begin as soon as the fracture callous starts bridging the fracture $[2,11,13]$. An unsuccessful outcome in foals may be the result of infection and non-union or be secondary to complications, such as cast sores.

Non-steroidal anti-inflammatory drugs are commonly administered to horses for analgesia and for their inhibitory effects on inflammation and edema [10]. They are indicated in horses with inflammatory conditions of the musculoskeletal system. Phenylobutazone and flunixin meglumine are the most frequently used non-steroidal anti-inflammatory drugs (NSAID) [10], though, naproxen and ketoprofen appear to have low toxicity in horses and a wide margin of safety [8]. Therefore, the latter two are recommended for foals rather than phenylobutazone 
or flunixin meglumine [8]. Gastrointestinal ulceration and renal papillary necrosis associated with NSAIDs are believed to occur secondary to decreased prostaglandin E2 synthesis [10]. Breed, age, and individual differences may exist in the susceptibility to NSAID toxicity [10]. Dosage, interval and route of administration for NSAIDs vary. The pharmacokinetics of the drugs in foals could be different to those in mature horses. Neonatal horses have less ability to eliminate phenylobutazone than adult horses after single IV-administered doses [10], and foals appear to be predisposed to the development of gastrointestinal ulcers [10]. Based on the arguments made earlier, the foal was put on ketoprofen in order to alleviate pain and to control oedema formation and inflammation.

In order to avoid orthopedic infections, consideration must be given to both gram-positive and gram-negative organisms [3]. Even in closed fractures, antibiotics are given prophylactically to suppress proliferation of exogenous organisms deposited in deeper tissues. Penicillin and aminoglycosides are currently a popular first choice in the treatment of equine infections. In the present case the choice of penicillin was based on the fact that it is bacteriocidal and acts directly on bacterial cell-wall synthesis and is highly active against many gram-positive organisms [9]. Streptomycin is, also, bacteriocidal and exerts its effect by inhibiting bacterial protein synthesis [9].

The prognosis for this foal was poor and there was concern for future complications at the growth plate of the third metacarpal bone. Although the prognosis was poor, the cost of treatment was relatively low, healing was rapid and uncomplicated and the foal returned to the paddock, healthy. The only relatively serious complication was the dropped fetlock, which was addressed successfully with regulated exercise.

\section{References}

1. Baxter, G.M., and Turner, A.S. 2002. Diseases of bone and related structures. pp. 401-407. In: Adams' Lameness in Horses, 5th ed. (Stashak, T.S. ed.), Lippincott Williams and Wilkins, Philadelphia.
2. Bertone, A.L. 2002. Fractures of the third metacarpal/metatarsal bone. pp. 811-816. In: Adams' Lameness in Horses, 5th ed. (Stashak, T.S. ed.), Lippincott Williams and Wilkins, Philadelphia.

3. Brown, M.P. 1998. Antibiotic therapy. pp. 21-25. In: Current Techniques in Equine Surgery and Lameness, 2nd ed. (White, N.A., and Moore, J.N., eds.), W.B. Saunders, Philadelphia.

4. Butler, J.A., Colles, C.M., Dyson, S.J., Kold, S.E., and Poulos, P.W. 1993. Fractures. In: Clinical Radiology of the Horse, Blackwell Science, Oxford.

5. Denny, H.R. 1996. First aid and external coaptation. pp. 4-8. In: Treatment of Equine Fractures, Wright, London.

6. Denny, H.R. 1996. Growth disturbances in foals. pp. 14-16. In: Treatment of Equine Fractures, Wright, London.

7. Denny, H.R. 1996. Fractures of 3rd metacarpus and 3rd metatarsus. pp. 73-76. In: Treatment of Equine Fractures, Wright, London.

8. Goodrich, L.R., and Nixon, A.J. 2006. Medical treatment of osteoarthritis in the horse. Vet. J. 171: 51-69

9. Markel, M.D. 1996. Bone fracture and the response of bone to stress. pp. 3-7. In: Equine Fracture Repair (Nixon, A.J. ed.), W.B. Saunders, Philadelphia.

10. Moore, R.M. 1998. Nonsteroidal antiinflammatory drugs. pp. 25-30. In: Current Techniques in Equine Surgery and Lameness, 2nd ed. (White, N.A., and Moore, J.N. eds.), W.B. Saunders, Philadelphia.

11. Nixon, A.J. 1996. General consideration in selecting cases for fracture repair. pp. 30-34. In: Equine Fracture Repair (Nixon, A.J. ed.), W.B. Saunders, Philadelphia.

12. Richardson, D.W. 1999. The metacarpal and metatarsal bones. pp. 810-819. In: Equine Surgery, 2nd ed. (Auer, J.A., and Stick, J.A. eds.), W.B. Saunders, Philadelphia.

13. Schneider, R.K., and Jackman, B.R. 1996. Fractures of the third metacarpus and metatarsus. pp. 179-194. In: Equine Fracture Repair (Nixon, A.J. ed.), W.B. Saunders, Philadelphia. 